\title{
Patient-centered rehabilitation of single, partial, and complete edentulism with cemented- or screw-retained fixed dental prosthesis: The First Osstem Advanced Dental Implant Research and Education Center Consensus Conference 2017
}

\author{
Marco Tallarico ${ }^{1}$, Marco Caneva ${ }^{2}$, Nicola Baldini ${ }^{3}$, Fulvio Gatti ${ }^{4}$, Marco Duvina ${ }^{5}$, \\ Mauro Billi ${ }^{6}$, Gaetano lannello ${ }^{7}$, Giacomo Piacentini ${ }^{8}$, Silvio Mario Meloni ${ }^{9}$, Marco Cicciù $^{10}$
}

Correspondence: Dr. Marco Cicciù

Email: acromarco@yahoo.it, mcicciu@unime.it

\author{
'Deparment of Medical Sciences, School of Dentistry, \\ Aldent University, Tirana, Albania, \\ ${ }^{2}$ ARDEC Academy, Ariminum Odontologica, Rimini, \\ Italy, \\ ${ }^{3}$ Department of Periodontics and Implantology, \\ University of Siena, Siena, Italy, \\ ${ }^{4}$ Department of Biomedical, Surgical and Dental \\ Sciences, Unit of Oral Surgery, San Carlo and San Paolo \\ Hospital, University of Milan, Milan, Italy, \\ ${ }^{5}$ Department of Oral Surgery and Implantology, \\ University of Florence, Florence, Italy, \\ ${ }^{6}$ Private Practice, Montevarchi, Italy, \\ ${ }^{7}$ Department of Biomedical and Dental Sciences and \\ Morphofunctional Imaging, School of Dentistry, Policlinic \\ "Gaetano Martino" University of Messina, Messina, Italy, \\ ${ }^{8}$ Private Practice, Pavia, Italy, \\ ${ }^{9}$ Department of Surgical Microsurgical and Medical \\ Science, University of Sassari, Sassari, Italy, \\ ${ }^{10}$ Department of Biomedical and Dental Sciences \\ and Morphological and Functional Imaging, Messina \\ University, Messina, Italy
}

\section{ABSTRACT}

The aim of this consensus conference was to provide clinical guidelines, based on the available evidence and on the author's daily practice and experience, for general dentistry and dental practitioners to allow them to delivery long-term successful restorations. Three groups of expert clinicians and dental technicians were invited to evaluate all of the scientific literature from 1967 up to March 2017 to identify relevant studies on assigned topics and to prepare in advance narrative/systematic review, written according to the Preferred Reporting Items for Systematic Reviews and Meta-Analyses guidelines, to fulfill the consensus statement criteria. The three topics assigned to the three groups were abutment/framework materials and customization (metal vs. metal-free restorations),

This is an open access journal, and articles are distributed under the terms of the Creative Commons Attribution-NonCommercial-ShareAlike 4.0 License, which allows others to remix, tweak, and build upon the work non-commercially, as long as appropriate credit is given and the new creations are licensed under the identical terms.

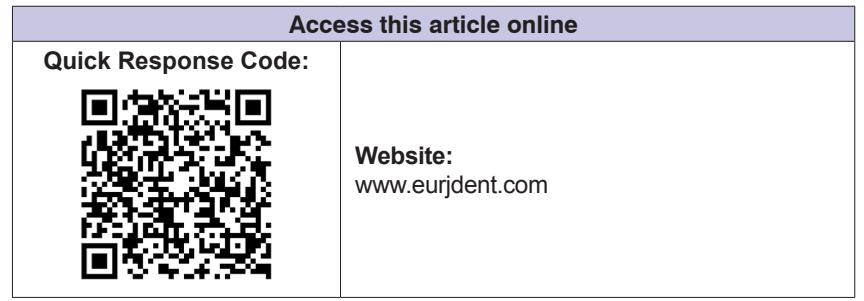

For reprints contact: reprints@medknow.com

How to cite this article: Tallarico M, Caneva M, Baldini N, Gatti F, Duvina M, Billi M, et al. Patient-centered rehabilitation of single, partial, and complete edentulism with cemented- or screw-retained fixed dental prosthesis: The First Osstem Advanced Dental Implant Research and Education Center Consensus Conference 2017. Eur J Dent 2018;12:617-26.

DOI: $10.4103 /$ ejd.ejd_243_18 
abutment/framework protocols and designs, and abutment/framework retentions (cemented- vs. screw-retained implant-supported prostheses). All the expert clinicians presented their results, and the lectures were followed by discussions. No significant differences in clinical parameters (marginal bone loss, bleeding on probing, and pocket probing depth) between screw- or cemented-retained were found for single and multiple implant-supported restorations. There is moderate evidence that nonoriginal abutments provide worse mechanical behavior than originals and high evidence that different implant neck designs do not offer any clinical or radiographic advantage. All the participants agreed that it is desirable to connect and remove abutments as few times as possible. There is medium evidence that an adequate platform switching tends to enhance tissue volume and stability in the medium- and long-term follow-up. No statistically significant differences exist between metal and zirconia as a framework material. The authors discussed and all agreed that retrievability and patient's expectation (function and esthetics) should guide the choice of the most adequate technique, component, and material.

Key words: Cemented-retained, dental implant prosthesis, screw-retained

\section{INTRODUCTION}

Implant-supported fixed dental prostheses (FDPs) are a predictable treatment, which allows to improve function and esthetics in partially and fully edentulous patients. ${ }^{[1]}$

Among the advantages of implant treatment, there is the possibility to apply an FDP for single and multiunit replacement without grinding the neighboring teeth and to have an FDP where formerly a removable denture would have been placed. However, the long-term outcome is one factor guiding a clinician whether implant treatment or conventional FDPs or removable partial dentures.

The third EAO consensus conference of 2012 focused on implant survival and complications of single crowns and FDPs, partial and complete. Based on prospective and retrospective cohort studies, the 10-year survival of conventional tooth-supported FDPs was 89.2\% compared to $86.7 \%$ for implant-supported FDPs. Despite high survival, biological and technical complications were frequent too and were reported to be higher than for implant-supported FDPs $(38.7 \%$ compared to $15.7 \%$ for conventional FDPs). The increased incidence of technical complications, such as veneer fractures, abutment rotation and breakage, abutment and screw loosening, and loss of retention for implant-supported FDPs, could be explained by the type of screw material, preload, abutment material, and connection configuration. ${ }^{[2,3]}$ Furthermore, the biologic complications such as increased microleakage, gingivitis, and marginal bone loss (MBL) have been reported to result from a poorly adapted implantabutment interface (IAI) ${ }^{[4-6]}$ However, there is still a lack of knowledge among clinicians regarding mechanical behavior and marginal accuracy of the implant-abutment complex and its relationship with technical complications as well as peri-implant bone loss (biologic complication).

Intwo-pieceimplants, microgapsat theIAIareunavoidable and are the consequences of the microtolerances between components required during the industrial manufacturing. ${ }^{[7]}$ The presence of this microgap allows microorganisms to proliferate close to the epithelial attachment, which often results in bone resorption up to $2 \mathrm{~mm}$ apical to the microgap. ${ }^{[5]}$ In addition to infection caused by plaque and its by-products, numerous etiological factors have been recognized to be specifically associated with peri-implantitis. ${ }^{[8]}$ Plaque-induced and prosthetically and surgically triggered peri-implantitis have been found to be different entities associated with distinguishing predictive profiles. ${ }^{[9]}$ Prosthetically triggered peri-implantitis was defined to be associated with plaque together with clinically evaluated cement remnants or together with incorrect finishing line positioning with respect to soft tissue, presence of overloading, crown ceramic chipping, abutment unscrewing (detected using periapical X-ray), and implant crack or fracture (detected at the time of abutment removal). ${ }^{[9]}$

The aim of this consensus conference was to provide clinical guidelines for general dentistry and dental practitioners to allow them to deliver long-term successful restorations. The prosthetic variables and the incidence of technical and biological complications were analyzed to recommend a prosthetic procedure as a standard for implant-supported rehabilitations, based on the available evidence and on the author's daily practice and experience.

\section{MATERIALS AND METHODS}

The first Osstem Advanced Dental Implant Research and Education Center (AIC) "close/consensus meeting" 
was held in September 2017 at the Micerium S.p.A., Avegno (Ge), Italy. Dr. Marco Tallarico, the Research Project Manager of Osstem AIC Italy, was the scientific responsible of the consensus meeting, and Micerium was responsible for the organization [Figure 1]. The Osstem AIC is a professional organization in implant dentistry. Outside funding was utilized to cover the travel costs of the participants. No other costs were provided for conducting the conference or for publishing the present results.

Three groups of expert clinicians and expert dental technicians were invited to evaluate all the scientific literature from 1967 up to March 2017 to identify relevant studies on the assigned topics and to prepare in advance narrative/systematic review to fulfill the consensus statement criteria. The following electronic databases were consulted: PubMed database of the US National Library of Medicine, Embase (Excerpta Medica database), and the Grey Literature Database (New York Academy of Medicine Grey Literature Report). The systematic reviews were written according to thePreferred Reporting Items for Systematic Reviews and Meta-Analyses guidelines (http:/ / www.prisma-statement.org). Quality assessment of selected full-text articles was performed according to the ARRIVE and CONSORT statement guidelines.

\section{Assigned topics and group discussion}

- Work Group 1: Abutment/framework retentions (cemented- vs. screw-retained implant-supported prostheses). Authors: Nicola Baldini, Gaetano Iannello, and Marco Duvina

- Work Group 2a: Abutment/framework designs: original versus nonoriginal abutments;

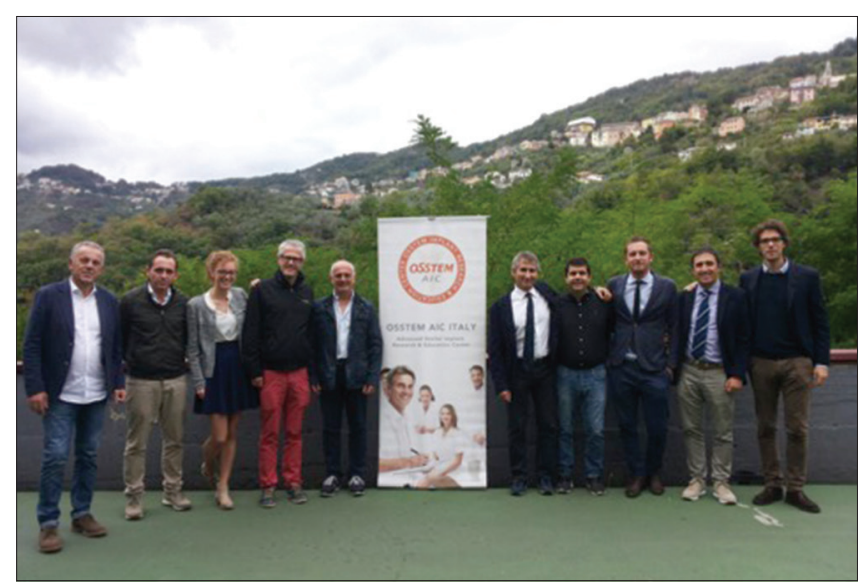

Figure 1: Osstem Advanced Dental Implant Research and Education Center participants. From left to right: Mauro Billi, Marco Duvina, Martina Caneva, Nicola Baldini, Gaetano Iannello, Ottaviano Miceli, Marco Tallarico, Fulvio Gatti, Silvio Mario Meloni, and Giacomo Piacentini macroarchitecture of the implant neck (implant shoulder design); and IAI. Authors: Marco Tallarico, Silvio Mario Meloni, and Martina Caneva

- Work Group 2b: Abutment/framework protocols: Abutment insertion protocol, such as one-abutment/one-time and platform switching. Authors: Marco Tallarico, Silvio Mario Meloni, and Martina Caneva

- Work Group 3: Abutment/framework materials and customization (metal vs. metal-free restorations). Authors: Fulvio Gatti, Mauro Billi, and Giacomo Piacentini.

The objective of the literature reviews of the different topics was to determine whether a procedure could be recommended as a standard based on the available evidence and on the author's daily practice and experience. The description of the technical aspects, advantages or disadvantages of each procedure, technical and biological complications and their management, esthetic outcomes, marginal bone level changes, costs, and retractability had to be addressed by each group. The clinicians had 3 months to complete the reviews, and all manuscripts had to be submitted to each participant at least 1 month before the consensus.

During the first part of the consensus, all the expert clinicians presented their results, and the lecture was followed by a first discussion. Similar topics of the reviews, with insufficient clinical data to be defined as subjects, were used to stimulate discussion among the consensus conference participants. During the second part, presentations were discussed step-by-step by all the participants, starting from the proposed variables. Some questions were answered by vote from the participants, and conclusions were provided.

\section{Inclusion criteria}

- Written in English

- Evaluate in their protocol the influence of the different analyzed variables on soft and hard tissue levels around single or multiple implants, as well as biological or mechanical complications

- In vivo randomized controlled clinical trials of implants $\geq 1$ year in function

- In vivo case-control studies of implants $\geq 1$ year in function

- In vivo prospective and retrospective cohort studies of implants $\geq 1$ year in function

- In vivo cross-sectional studies $\geq 1$ year in function

- In vitro studies 
- Systematic reviews, narrative reviews, and consensus statements.

\section{Exclusion criteria}

- Observational (prospective or retrospective) cohort studies without control group

- Finite element analyses

- Animal studies

- Reports with $<5$ cases

- Reports involving mini-implants, zirconia implants, or blade implants

- Clinical reports on implants $<1$ year in function

- Abstracts, commentaries, or editorials.

\section{Terms definitions}

According to the latest Glossary of Prosthodontic Terms ${ }^{[10]}$ and Mesh terms (https://www.ncbi.nlm. nih.gov/mesh):

- Retention: The quality inherent in the implant-supported dental prosthesis acting to resist the forces of dislodgement along the path of placement. The fixation of an implant reconstruction can either be accomplished by screw retaining the reconstruction on the implant or on a screw onto implant abutment or by cementing the reconstruction on standardized or customized abutments, in the same manner as luting an FDP to natural teeth

- Original abutment: An abutment produced by the same implant manufacturer based on original nominal dimensions. Itcan be stock (or standardized) or computer-aided design/computer-aided manufacturing (CAD/CAM)

- Nonoriginal abutment: An abutment produced by a different implant manufacturer (a third-party company). It can be certified or compatible. Nonoriginal certified abutment is an abutment produced by a third-party milling center, directly or indirectly certified for certain implant companies. It can be gold cast or CAD/CAM. Nonoriginal compatible abutment is an abutment produced by an implant company compatible with other implant systems with which has the same IAI. It might also be manufactured by a generic producer not directly involved in the implant manufacturing. It can be stock or CAD/CAM

- Macroarchitecture of the implant neck: Dental implants come in a variety of designs to adapt to the individual patient situation as well as the preferences of the dentist. Regarding the implant neck design, there is a choice between two-piece (conventional flat platform neck design with the same level on the $360^{\circ}$ ), one-piece (implant body and abutment are manufactured as one piece), and scalloped or sloped implants (implant neck designs aimed to mirror the natural alveolar ridge curvature)

- Dental abutment: A tooth, tooth root, or implant component that serves as support and/or retention for a dental prosthesis ([anatomic] healing abutment; angled/angulated abutment; CAD/CAM abutment; castable abutment; ceramic/metal abutment; straight abutment; rotating/nonrotating abutment; prefabricated/ stock abutment; preparable abutment; standard abutment; temporary abutment; transmucosal abutment; and Universal Clearance Limited Abutment UCLA abutment)

- One-abutment/one-time: Definitive abutments placed at implant insertion and never removed

- IAI: Common contact surface area between an implant abutment and the supporting implant

- Platform switching: A design strategy including the connection of a smaller diameter abutment relative to the platform diameter of the titanium implant.

\section{RESULTS}

In the effort to determine whether a prosthetic procedure could be recommended as a standard for implant-supported rehabilitations, based on the available evidence and on the author's daily practice and experience, a small national and independent study group made up of nine established clinical experts was formed. Their collective long-term clinical and research experience with implant-supported restorations would provide scope for a prudent and objective synthesis of the relevant available literature and of the clinical concerns related to this topic. Based on the literature reviews, the participants of each group prepared consensus statements, clinical recommendations, and implications related to their topic in written form that were approved during the plenary session (second part of the consensus). These statements are presented here in a condensed version. The keywords used to search the literature and the number of final articles from which data are extracted, as described in Table 1.

Work Group 1: Abutment/framework retention (cemented- vs. screw-retained implant-supported prostheses)

A total of 245 potentially relevant titles and abstracts were found after the electronic and manual search. After the first and second stage of selection, a total of 40 manuscripts were selected. Of these, three were randomized controlled trials (RCTs), eight were 
Tallarico, et al.: Osstem dental implant consensus conference

\begin{tabular}{|c|c|}
\hline Topic & Search strategy \\
\hline \multicolumn{2}{|c|}{$\begin{array}{l}\text { Cemented versus screw-retained } \\
\text { implant-supported prostheses }\end{array}$} \\
\hline $\begin{array}{l}\text { Original versus nonoriginal } \\
\text { abutments }\end{array}$ & $\begin{array}{l}\text { ((“Dental Implant-Abutment Design" [Mesh] OR "Dental Implant-Abutment" OR "Dental Implant } \\
\text { Abutment") AND (“Computer-Aided Design"[Mesh] OR “*original” OR “compatible" OR "avatar")) }\end{array}$ \\
\hline $\begin{array}{l}\text { Macroarchitecture of } \\
\text { the implant neck }\end{array}$ & $\begin{array}{l}\text { (((“"Dental implants"[Mesh] AND "dental implant abutment design”[Mesh]) OR "dental implant abutment } \\
\text { interface"[All Fields]) OR (one[All Fields] AND piece[All Fields] AND implant[All Fields])) OR ((“scalloped”[All } \\
\text { Fields]) AND implant[All Fields]) OR (sloped[All Fields] AND implant[All Fields]) AND English[lang]) }\end{array}$ \\
\hline Implant abutment interface & $\begin{array}{l}\text { ("dental implants" [MeSH] AND "bacterial contamination" OR "presence of bacterium" OR "dental } \\
\text { leakage/microbiology" [MeSH] OR "microleakage" OR "microbiological findings" OR "microbiological } \\
\text { colonization" OR "microbiota" OR "peri-implant microflora" AND "peri-implantitis" [MeSH] OR "peri-implant } \\
\text { pathology" OR "peri-implant disease" AND "Dental Abutments*/microbiology" [MeSH]"connection, } \\
\text { implant-abutment" OR "dental Implant-abutment design" [MeSH] OR "implant-abutment junction" OR } \\
\text { "implant-abutment microgap" OR "inner space of dental implants" OR "inner part of dental implants") }\end{array}$ \\
\hline Abutment insertion protocol & $\begin{array}{l}\text { (((((Dental Implant-Abutment*[Title/Abstract]) OR One-abutment*[Title/Abstract]) OR } \\
\text { abutment dis*[Title/Abstract]) AND (Immediate Dental Implant Loading[Title/Abstract]) } \\
\text { OR Immediate loading[Title/Abstract]) OR Immediate abutment[Title/Abstract]) }\end{array}$ \\
\hline Platform switching & 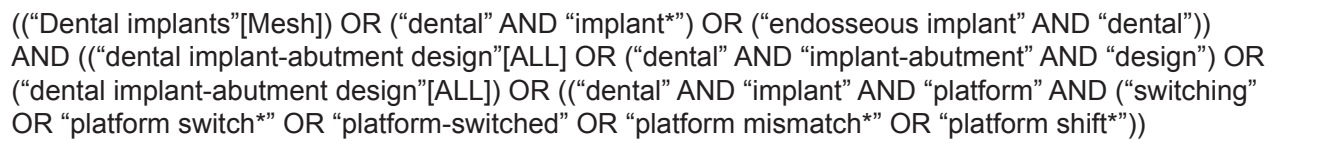 \\
\hline $\begin{array}{l}\text { Metal versus metal-free } \\
\text { restorations }\end{array}$ & $\begin{array}{l}\text { ((“Dental Implants"[Mesh]) AND (“Dental Prosthesis, Implant-Supported”[Mesh] OR "Denture, Partial, } \\
\text { Fixed"[Mesh] OR "Dentures"[Mesh]) AND (“Complications" OR "Survival”)) }\end{array}$ \\
\hline
\end{tabular}

observational comparative studies (3 retrospective and 5 prospective), 12 were systematic review of the literature, and 17 were in vitro studies. Based on the selected studies, all the participants agreed that implant-supported FDPs can be connected either by screw retention or cement retention. However, when using a cemented protocol, extreme care must be taken to avoid residual cement. In general, screw-retained restorations are suggested because they are easier to remove and no cement is required. Nevertheless, they present more technical complications (screw loosening, chipping of the veneering material). To reduce complications, skills of the clinician and the dental technician are required.

All the participants agreed that prosthetic screws have to be tight at the recommended torque. A second-time tightening (10 min later) is recommended to reduce the risk of screw loosening. It is suggested that the dental technician uses the same driver at the same torque of the clinician. There is still lack of evidence regarding the efficacy of sealing agents on preload maintenance of screw-retained implant-supported prostheses. Screw-retained restoration can be either with one or two prosthetic screws (if multiple abutments have been used). Advantages of multiple abutments are one abutment at one-time concept, correction of disparallelism between implants, and move inflammation at tissue level. Disadvantages are only $15 \mathrm{Ncm}$ of prosthetic torque, wide diameter for esthetics, and reducing vertical space for restorations.
In case of cemented-retained restorations, the most important complications are biological consequence of cement-retained and fracture of the prosthetic screw due to screw loosening. ${ }^{[11]}$

Single or multiple, cemented-retained, metal-free, lithium disilicate restorations could be cemented on abutments or frameworks, out of the patients' mouth. Analyzed data reported that single implant-supported restoration can be either screw- or cemented-retained, with no significant differences in clinical parameters (MBL, bleeding on probing [BOP], and pocket probing depth). Participants agreed that the final choice can be made based on esthetics, tissue biotype, interarches space, implant disparellelism, and personal expertise.

Similarly, multiple restorations can be either screw- or cemented-retained. In case of partial restorations, authors reported no differences between screw- and cemented-retained FDPs. Again, the choice can be made based on esthetics, tissue biotype, interarche space, and implant disparellelism. All the participants agreed that CAD/CAM abutments and/or frameworks should be preferred to improve esthetics and reduce potential biological complications, in both single and multiple restorations.

In case of complete restorations, the most frequent technical complications were screw fracture and chipping or fracture of the veneering material. ${ }^{[12]}$ To reduce complications, an adequate number of 
implants and their correct position are recommended. In fact, in case of multiple, implant-level restorations, the IAI works as a flat-to-flat interface. Long-span, implant-supported FDPs should be at abutment level (screwed on multi-abutment or cemented on stock or customized abutment). The longer the span is, the greater the distortion and subsequently the misfit that may occur. Hence, in case of complete restorations, the use of multi-abutments is strongly suggested. Disadvantages of multi-abutments are wide emergence profile, maximum $15 \mathrm{Ncm}$ of torque, and reduced interarch space for the prosthesis. Otherwise, the smaller the inter-implant distance, the lower is the risk of prosthetic misfit. Hence, short-span fixed partial prostheses can be at implant level. Implants parallelism is mandatory.

Work Group 2a: Abutment/framework design: Original versus nonoriginal abutments; macroarchitecture of the implant neck (implant shoulder design); implant-abutment interface

Original versus nonoriginal abutments

After second-step selection and manual research, 16 full texts were included. Overall, data from 255 original abutments (120 stock and 135 CAD/CAM) and 364 nonoriginal abutments (211 stock and 153 CAD/CAM) were analyzed.

There is moderate evidence from in vitro studies that nonoriginal abutments provide worse mechanical behavior than originals. Nevertheless, certified abutment with high-quality control seems to have similar outcomes than original. Compatible abutments should not be used. Furthermore, little but positive evidence is present on angled screw-channel solutions; nevertheless, they do not change implants' disparallelism. ${ }^{[13]}$

Macroarchitecture of the implant neck

After electronic and manual search, 16 manuscripts were selected. Of these, seven were RCTs, four were observational comparative studies (2 retrospective and 2 prospective), and five were systematic review of the literature. Overall, 221 one-piece implants were placed in 107 patients, 139 scalloped implants placed in 96 patients, and 366 flat implants (same level on $360^{\circ}$ ) placed in 207 patients. No comparative studies reporting data on sloped implants that fulfilled the inclusion criteria were found in this research.

There is high evidence that different implant neck designs do not offer any clinical or radiographic advantage. ${ }^{[14]}$ Hence, it is preferable to have a two-piece implant with convergent or straight neck design, with the same level on $360^{\circ}$. Nevertheless, other factors related to implant neck design, such as the use of microthreads and modifications in implant surface characteristics, could preserve peri-implant bone. In addition, some authors recently demonstrated significantly higher MBL rates around implants with shorter compared with longer prosthetic abutments, concluding that the height of the abutment plays a critical role in the marginal bone maintenance in screw-retained prostheses, in spite of the platform-switching distance. ${ }^{[15]}$

\section{Implant-abutment interface}

After electronic and manual search, 14 manuscripts, including 1126 implants, were selected. Then, four in vitro studies and three in vivo RCTs were analyzed to find possible significant differences between internal and external connections.

Bacterial colonization at the IAI seemed to be unavoidable. ${ }^{[16]}$ Nevertheless, based on the selected studies, there is medium evidence that a stable connection is essential to reduce micromovements and microleakage. Stable implant-abutment connection depends on a several number of variables including, but not limited to, the accuracy (manufacturing) of the implant's components. In general, internal connections are considered to be more stable than external. However, external connections can provide prosthetic stability with the proper screw preload, and it could be suggested in complete restorations.

Work Group 2b: Abutment/framework protocols: Abutment insertion protocol; platform switching Abutment insertion protocol (one-abutment/one-time) After electronic and manual search, 14 manuscripts, including 535 patients with 994 implants, were selected. Of these, five were prospective cohort studies, three were retrospective cohort studies, and six were RCTs with a parallel group design that was included in the quantitative analysis. One-abutment one-time protocol has no clinical influence on the esthetic outcome. Results from systematic review and meta-analysis showed a reduction of $0.3 \mathrm{~mm}$ for MBL and $0.2 \mathrm{~mm}$ for buccal reduction. ${ }^{[17]}$ The main limitation of this protocol is that a cemented-retained restoration is required. Nevertheless, all the participants agreed that it is desirable to connect and remove abutments as few times as possible. 
Tallarico, et al.: Osstem dental implant consensus conference

Platform switching

After electronic and manual search, 12 manuscripts were selected for this consensus. Of these, three were RCTs by the authors of this consensus and six were systematic review with meta-analysis. Two were narrative reviews, and one was systematic review of the literature.

There is medium evidence that an adequate platform switching tends to enhance tissue volume and stability in the medium- and long-term follow-up. ${ }^{[18,19]}$ Nevertheless, all the participants agreed to apply the platform switching concept.

Work Group 3: Abutment/framework materials and customization: Metal versus metal-free restorations After electronic and manual search, 13 manuscripts were selected for this consensus. Based on RCTs, zirconia frameworks exhibited similar outcomes to metal-based FDPs. The majority of technical and biologic outcome measures were not significantly different. Chipping of the veneering material was the most frequent complication for both types of restorations. ${ }^{[20,21]}$

All the participants agreed that in the presence of a thin phenotype, all ceramic materials should be used for abutments and/or crowns. There is strong clinical evidence that when all ceramic materials are used, the use of a titanium base connector is advised. Regarding the production method, customized abutments can improve tissue support compared with stock abutments. In addition, customized abutments improve cement line position in cement-retained crowns, and hence, CAD/CAM abutments should be preferable. The accuracy of the workflow depends mainly on physical material properties (impression, master model, and casting) and human-related factors (timing and manual handling).

In the smile zone, a single, CAD/CAM zirconia abutment with a titanium base connector is advised. Metal-free materials are advised as veneering material (i.e., lithium disilicate). In the nonsmile zone, it is advisable to use a metal (titanium or $\mathrm{Cr}-\mathrm{Cb}$ ) abutment (titanium) with a porcelain fused to metal $(\mathrm{Cr}-\mathrm{Cb})$ crown, cemented on the top. Stock or CAD/CAM abutment can both be used. A custom UCLA-metal ceramic abutment or a customized CAD/CAM abutment can be screwed at implant level and stratified with porcelain. In this case, gold alloy or $\mathrm{Cr}-\mathrm{Cb}$ is recommended as an abutment/framework material. No statistically significant differences exist between metal and zirconia as a framework material.
Data from the literature showed that most chipping of the veneering materials is observed when using porcelain fused to zirconia. Nevertheless, all the participants agreed that these complications can be reduced with technical experience and following an accurate production protocol. Furthermore, participants highlighted that screw-retained restorations are easy to remove in case of complications. Monolithic solution in the posterior area and stratified restorations in the anterior could be another good solution to reduce technical complications; nevertheless, there is still lack of scientific evidence about monolithic reconstructions. For all of these reasons, all the participants agreed that prevention and management of complications should be improved.

No scientific evidence is present for the new prosthetic solutions: single, partial, or complete restorations bonded on metallic abutment; monolithic zirconia restorations; and metallic ring for zirconia-based, screw-retained restorations. There is also no evidence on dental composite as a restorative material (titanium-composite restoration).

\section{DISCUSSIONS}

It seems clear that, regardless of all the efforts to limit it, there are many factors causing MBL around dental implants. The reformation of biologic width around dental implants has been hypothesized as one of the most likely causes of early implant bone loss. ${ }^{[22]}$ Several factors, such as implant micro- and macro-design, as well as surgical and prosthetic aspects, may all contribute to this process. ${ }^{[23-25]}$ In many cases, adjunctive bone loss can be attributed to complications associated with the implant and its components such as loose of abutment screws, broken components, bacterial contamination of interfaces, and cement in the soft tissues. In fact, according to an observational study from Canullo et al., 30.4\% of peri-implantitis can be classified as prosthetically triggered..$^{[9]}$

According to the Seventh European Workshop on Periodontology, ${ }^{[8]}$ peri-implantitis was defined as positive BOP and/ or suppuration, in combination with radiographic $\mathrm{MBL} \geq 2 \mathrm{~mm} \cdot{ }^{[8]}$ In a recent observational study, Galindo-Moreno et al. found that implants with increased MBL rates during the immediate postloading healing period are more likely to reach MBL values that may compromise their mid-long-term success and survival rates. The cutoff value suggested from this study was $2 \mathrm{~mm}$ at 18 months. Factors influencing 
MBL during this healing period are smoking, bone, age, connection type, and plaque accumulation. ${ }^{[26]}$ An open-cohort prospective study reported patients receiving bone level implants with a rough surface (Ra of $2.5 \sim 3.0 \mu \mathrm{m}$ ) sandblasted with alumina and acid-etched and featuring an internal hex and $11^{\circ}$ conical connection (Osstem TSIII, XXX). ${ }^{[27]}$ Following the results of this study, a physiological marginal bone remodeling of $0.37 \mathrm{~mm}$ within 1 year after loading can be expected in the daily practice.

It is well known that implant-supported FDPs are a safe and predictable treatment method with high survival rates. However, biological and technical complications are frequent (33.6\%). ${ }^{[1,28,29]}$ A recent systematic review by Pjetursson et al. suggested that a way to minimize the incidence of complications is to choose reliable components and materials. ${ }^{[1]}$ Moreover, the patients should be placed in well-structured maintenance systems immediately after treatment.

During this consensus conference, in the effort to determine whether a prosthetic procedure could be recommended as a standard for implant-supported rehabilitations, the authors discussed and all agreed that retrievability and patient's expectation (function and esthetics) should guide the choice of the most adequate technique, component, and material.

Although screw-retained reconstructions exhibit more technical problems compared with cemented prostheses, screw-retained reconstructions are more easily retrievable and show less biological complications (implant loss, bone loss $>2 \mathrm{~mm}$ ). Furthermore, eventual technical and/or biological complications can be treated more easily. ${ }^{[12]}$ At the same time, especially for posterior regions and/or in case of reduced mouth opening, this prosthetic solution would make easier and faster the tightening procedure. However, screw-retained restorations require a careful prosthetically driven planning, performed according to the esthetic and functional needs, which could be achieved using a computer-assisted template-guided implant placement. These approaches also potentially reduce the risks for implant and prosthetic long-term failure. ${ }^{[30]}$ Moreover, an angulated screw channel abutment may allow to further compensate divergence up to $25^{\circ}$, in order not to affect esthetics.

Stability of the peri-implant soft and hard tissues is prerequisites for a long-term esthetic and function of implant-supported restoration. In the last year, new implant and abutment designs have been proposed to minimize the crestal bone loss. Platform switching has been proposed as an effective prosthetic concept to reduce the amount of peri-implant bone loss around dental implants. ${ }^{[20]}$ On the contrary, there was sufficient evidence that implants with scalloped or sloped marginal contour offer no benefit when compared to two-piece, conventional flat implants. ${ }^{[14]}$

If possible, repeated abutment dis/re-connections may be avoided due to significantly increased MBL and buccal recession. ${ }^{[17]}$ Placing a definitive abutment the day of the surgery presupposes that the definitive restoration will be cemented above. Otherwise, screwing multiunit abutments immediately on the day of the surgery (i.e., complete restorations) allow to deliver a screw-retained restoration maintaining advantages of the one-abutment one-time concept.

Standardized titanium abutments still represent, worldwide, the gold standard for implant reconstructions due to the good long-term success rates reported in clinical studies. ${ }^{[31-37]}$ Nevertheless, these abutments exhibit several shortcomings. First, metallic abutments can provide grayish color when in contact with thin soft tissues which may lead to esthetic problems. ${ }^{[31]}$ Second, to develop a natural emergence profile of the reconstruction, the standardized shape needs to be modified directly by a tooth-like shape of the crown. This situation may lead to over-contouring the reconstruction. Finally, the standardized height of the crown margin may impair the removal of the excess cement, leading to possible biological complications. To overcome these problems, customized CAD/CAM abutments have been developed. These may be made of either titanium or zirconia. It has been shown that zirconia abutments exhibited the same survival and a similar esthetic outcome as titanium abutments. ${ }^{[35-40]}$ Moreover, zirconia abutments allow to improve esthetics leading to customized emergence profile and cement line. One limitation of the zirconia abutments may be that their material strength is lower than that of titanium, and hence, all the participants agreed that a metallic ring for zirconia-based, screw-retained restorations is mandatory. Because of their greater optical properties and esthetic results, the use of zirconia abutments is suggested for smile regions even if more studies should be performed to longitudinally analyze biological response.

\section{CONCLUSIONS}

Expert opinion and available evidence lead the consensus group to conclude that: 
- Crowns/bridges can be connected to implants either by screw retention or cement retention. When using a cemented protocol, extreme care must be taken to avoid residual cement. A customized abutment is recommended

- Screw-retained restorations are suggested because they are easier to remove. Prosthetic screws have to be tight at the recommended torque

- Long-span, implant-supported, FDPs should be at abutment level (screwed on multi-abutments or cemented on stock or customized abutments)

- A stable connection is essential to reduce micromovements and microleakage, and different implant neck designs do not offer any clinical or radiographic advantage. However, it is desirable to connect and remove abutments as little as possible

- Compatible abutments should not be used. Certified abutment with high-quality control seems to have similar outcomes than original

- There is medium evidencethat an adequate platform switching tends to enhance tissue volume and stability in the medium- and long-term follow-up

- Zirconia frameworks exhibited similar outcomes to metal-based FDP. Chipping of the veneering material was the most frequent complication for both types of restorations. Nevertheless, in the smile zone and in the presence of a thin phenotype, all ceramic materials should be used for abutments and/or crowns

- No scientific evidence is present for new prosthetic solutions: single, partial, or complete restorations bonded on metallic abutment; monolithic zirconia restorations; metallic ring for zirconia-based, screw-retained restorations; and dental composite as a restorative material (titanium-composite restoration). Well-designed RCT should be conducted.

It is recognized that many of the clinical recommendations suggested by the consensus group are not yet associated with strong evidence. Readers should note that the experience of the group was used in formulating the recommendations.

\section{Acknowledgments}

Micerium SPA supported the authors' travel expenses and hosted the meeting. Nevertheless, the authors did not receive any financial reward. We are grateful to Dr. Martina Caneva for her valuable support during the meeting and for her critical revision of the manuscript.

\section{Financial support and sponsorship}

Micerium SPA supported the authors' travel expenses and hosted the meeting.

\section{Conflicts of interest}

There are no conflicts of interest.

\section{REFERENCES}

1. Pjetursson BE, Thoma D, Jung R, Zwahlen M, Zembic A. A systematic review of the survival and complication rates of implant-supported fixed dental prostheses (FDPs) after a mean observation period of at least 5 years. Clin Oral Implants Res 2012;23 Suppl 6:22-38.

2. Tsuge T, Hagiwara Y. Influence of lateral-oblique cyclic loading on abutment screw loosening of internal and external hexagon implants. Dent Mater J 2009;28:373-81.

3. Vigolo P, Fonzi F, Majzoub Z, Cordioli G. Evaluation of gold-machined UCLA-type abutments and CAD/CAM titanium abutments with hexagonal external connection and with internal connection. Int J Oral Maxillofac Implants 2008;23:247-52.

4. Broggini N, McManus LM, Hermann JS, Medina RU, Oates TW, Schenk RK, et al. Persistent acute inflammation at the implant-abutment interface. J Dent Res 2003;82:232-7.

5. Hermann JS, Schoolfield JD, Schenk RK, Buser D, Cochran DL. Influence of the size of the microgap on crestal bone changes around titanium implants. A histometric evaluation of unloaded non-submerged implants in the canine mandible. J Periodontol 2001;72:1372-83.

6. King GN, Hermann JS, Schoolfield JD, Buser D, Cochran DL. Influence of the size of the microgap on crestal bone levels in non-submerged dental implants: A radiographic study in the canine mandible. J Periodontol 2002;73:1111-7.

7. Abrahamsson I, Berglundh T, Lindhe J. The mucosal barrier following abutment dis/reconnection. An experimental study in dogs. J Clin Periodontol 1997;24:568-72.

8. Lang NP, Berglundh T; Working Group 4 of Seventh European Workshop on Periodontology. Periimplant diseases: Where are we now? - Consensus of the seventh European workshop on periodontology. J Clin Periodontol 2011;38 Suppl 11:178-81.

9. Canullo L, Tallarico M, Radovanovic S, Delibasic B, Covani U, Rakic M. Distinguishing predictive profiles for patient-based risk assessment and diagnostics of plaque induced, surgically and prosthetically triggered peri-implantitis. Clin Oral Implants Res 2016;27:1243-50.

10. The glossary of prosthodontic terms: Ninth edition. J Prosthet Dent 2017;117:e1-105.

11. Linkevicius T, Vindasiute E, Puisys A, Linkeviciene L, Maslova N, Puriene A. The influence of the cementation margin position on the amount of undetected cement. A prospective clinical study. Clin Oral Implants Res 2013;24:71-6.

12. Sailer I, Mühlemann S, Zwahlen M, Hämmerle CH, Schneider D. Cemented and screw-retained implant reconstructions: A systematic review of the survival and complication rates. Clin Oral Implants Res 2012;23 Suppl 6:163-201.

13. Gatti F, Gatti C, Tallarico M, Tommasato G, Meloni SM, Chiapasco M. Maxillary sinus membrane elevation using a special drilling system and hydraulic pressure: A 2-Year prospective cohort study. Int J Periodontics Restorative Dent 2018;38:593-9. doi: 10.11607/prd.3403.

14. Tallarico M, Caneva M, Meloni SM, Xhanari E, Omori Y, Canullo L. Survival and success rates of different shoulder designs: A systematic review of the literature. Int J Dent 2018;2018:6812875. doi: 10.1155/2018/6812875. eCollection 2018. Review.

15. Galindo-Moreno P, León-Cano A, Monje A, Ortega-Oller I, O’ Valle F, Catena A. Abutment height influences the effect of platform switching on peri-implant marginal bone loss. Clin Oral Implants Res 2016;27:167-73.

16. Tallarico M, Canullo L, Caneva M, Özcan M. Microbial colonization at the implant-abutment interface and its possible influence on periimplantitis: A systematic review and meta-analysis. J Prosthodont Res 2017;61:233-41.

17. Tallarico M, Caneva M, Meloni SM, Xhanari E, Covani U, Canullo L, et al. Definitive abutments placed at implant insertion and never removed: Is it an effective approach? A systematic review and meta-analysis of randomized controlled trials. J Oral Maxillofac Surg 2018;76:316-24

18. Pozzi A, Tallarico M, Moy PK. Three-year post-loading results of a randomised, controlled, split-mouth trial comparing implants with different prosthetic interfaces and design in partially posterior 
edentulous mandibles. Eur J Oral Implantol 2014;7:47-61.

19. Canullo L, Caneva M, Tallarico M. Ten-year hard and soft tissue results of a pilot double-blinded randomized controlled trial on immediately loaded post-extractive implants using platform-switching concept. Clin Oral Implants Res 2017;28:1195-203.

20. Sailer I, Balmer M, Hüsler J, Hämmerle CHF, Känel S, Thoma DS, et al. Comparison of fixed dental prostheses with zirconia and metal frameworks: Five-year results of a randomized controlled clinical trial. Int J Prosthodont 2017;30:426-8.

21. Nicolaisen MH, Bahrami G, Schropp L, Isidor F. Comparison of metal-ceramic and all-ceramic three-unit posterior fixed dental prostheses: A 3-year randomized clinical trial. Int J Prosthodont 2016;29:259-64

22. Oh TJ, Yoon J, Misch CE, Wang HL. The causes of early implant bone loss: Myth or science? J Periodontol 2002;73:322-33.

23. Elian N, Bloom M, Dard M, Cho SC, Trushkowsky RD, Tarnow D. Effect of interimplant distance $(2$ and $3 \mathrm{~mm}$ ) on the height of interimplant bone crest: A histomorphometric evaluation. J Periodontol 2011;82:1749-56.

24. Klinge B. Peri-implant marginal bone loss: An academic controversy or a clinical challenge? Eur J Oral Implantol 2012;5 Suppl 1:S13-9.

25. Tarnow DP, Cho SC, Wallace SS. The effect of inter-implant distance on the height of inter-implant bone crest. J Periodontol 2000;71:546-9.

26. Galindo-Moreno P, León-Cano A, Ortega-Oller I, Monje A, O Valle F, Catena A. Marginal bone loss as success criterion in implant dentistry: Beyond 2 mm. Clin Oral Implants Res 2015;26:e28-34.

27. Tallarico M, Meloni SM. Open-cohort prospective study on early implant failure and physiological marginal remodeling expected using sandblasted and acid-etched bone level implants featuring an 11 Morse taper connection within one year after loading. J Oral Sci Rehabil 2017;3:68-79.

28. Albrektsson T, Donos N; Working Group 1. Implant survival and complications. The third EAO consensus conference 2012. Clin Oral Implants Res 2012;23 Suppl 6:63-5.

29. Pjetursson BE, Brägger U, Lang NP, Zwahlen M. Comparison of survival and complication rates of tooth-supported fixed dental prostheses (FDPs) and implant-supported FDPs and single crowns (SCs). Clin Oral Implants Res 2007;18 Suppl 3:97-113.

30. Tallarico M, Meloni SM. Retrospective analysis on survival rate, template-related complications, and prevalence of peri-implantitis of 694 anodized implants placed using computer-guided surgery: Results between 1 and 10 years of follow-up. Int J Oral Maxillofac Implants 2017;32:1162-71.

31. Linkevicius $\mathrm{T}$, Vaitelis J. The effect of zirconia or titanium as abutment material on soft peri-implant tissues: A systematic review and meta-analysis. Clin Oral Implants Res 2015;26 Suppl 11:139-47.

32. Henry PJ, Laney WR, Jemt T, Harris D, Krogh PH, Polizzi G, et al. Osseointegrated implants for single-tooth replacement: A prospective 5-year multicenter study. Int J Oral Maxillofac Implants 1996;11:450-5.

33. Cicciù M, Risitano G, Maiorana $C$, Franceschini G. Parametric Analysisof the strenght in the "toronto" osseous-prosthesis system. Minerva Stomatologica 2009;58:1-2; 9-23.

34. Scheller H, Urgell JP, Kultje C, Klineberg I, Goldberg PV, Stevenson-Moore P. A 5-year multicenter study on implant-supported single crown restorations. Int J Oral Maxillofac Implants 1998:13:212-8.

35. Sailer I, Zembic A, Jung RE, Siegenthaler D, Holderegger C, Hämmerle $\mathrm{CH}$. Randomized controlled clinical trial of customized zirconia and titanium implant abutments for canine and posterior single-tooth implant reconstructions: Preliminary results at 1 year of function. Clin Oral Implants Res 2009;20:219-25.

36. Cervino G, Romeo U, Lauritano F, Bramanti E, Fiorillo L, D'Amico C, et al. Fem and von mises analysis of OSSTEM ® dental implant structural components: Evaluation of different direction dynamic loads. Open Dent J 2018;12:219-29.

37. Bramanti E, Cervino G, Lauritano F, Fiorillo L, D'Amico C, Sambataro S. FEM and von mises analysis on prosthetic crowns structural elements: Evaluation of different applied materials. ScientificWorldJournal 2017;2017:1029574.

38. Cicciu M, Bramanti E, Matacena G, Guglielmino E, Risitano G. FEM evaluation of cemented-retained versus screw-retained dental implant single-tooth crown prosthesis. Int J Clin Exp Med 2014;7:817-25.

39. Lauritano F, Runci M, Cervino G, Fiorillo L, Bramanti E, Cicciù M. Three-dimensional evaluation of different prosthesis retention systems using finite element analysis and the von mises stress test. Minerva Stomatol 2016;65:353-67.

40. Cicciù M, Cervino G, Bramanti E, Lauritano F, Lo Gudice G, Scappaticci L. FEM analysis of mandibular prosthetic overdenture supported by dental implants: Evaluation of different retention methods. Comput Math Methods Med 2015;2015:943839. 